\title{
Relaxation of Coulomb States in Semiconductors Probed by FEL Radiation
}

\author{
$\underline{\text { R.Kh. Zhukavin }}^{1}$, K.A. Kovalevsky ${ }^{1}$, V.V. Tsyplenkov ${ }^{1}$, S.G. Pavlov ${ }^{2}$, H-W. Hübers ${ }^{2,3}$ \\ Yu.Yu. Choporova ${ }^{4,5}$, B.A. Knyazev ${ }^{4,5}$, J.M. Klopf ${ }^{6}$, B. Redlich ${ }^{7}$, N.V. Abrosimov ${ }^{8}$, Yu.A. \\ Astrov $^{9}$, V.N. Shastin ${ }^{1}$ \\ ${ }^{1}$ Institute for Physics of Microstructures, Nizhny Novgorod, Russia, zhur@ipmras.ru \\ ${ }^{2}$ Institute of Optical Sensor Systems, German Aerospace Center (DLR), Berlin, Germany \\ ${ }^{3}$ Department of Physics, Humboldt-Universität zu Berlin, Berlin, Germany \\ ${ }^{4}$ Budker Institute of Nuclear Physics, Novosibirsk, Russia \\ ${ }^{5}$ National Research Novosibirsk State University, Novosibirsk, Russia \\ ${ }^{6}$ Helmholtz-Zentrum Dresden-Rossendorf, Dresden, Germany \\ ${ }^{7}$ FELIX Laboratory, Radboud University, Institute of Molecules and Materials, Nijmegen, The Netherlands \\ ${ }^{8}$ Leibniz-Institut für Kristallzüchtung (IKZ), Berlin, Germany \\ ${ }^{9}$ Ioffe Institute, St. Petersburg, Russia
}

The typical semiconductors such as silicon and germanium with embedded donors and acceptors have been intensively studied since $1950^{\text {th }}$ [1]. The main reason was the importance of doped semiconductors in the production of various electronic devices based on the properties of $p-n$ junction or Schottky-junction. The new interest to Coulomb centers in semiconductors has arisen from several new directions including single-atom electronics, quantum logic and lasing based on impurity centers. For use of Coulomb centers in the frame of any of those fields one should know such properties of bound electron as energy spectrum and relaxation rates. The experimental methods being used for the investigations compose a very broad set of different techniques involving transport and optics and their combinations under various conditions. Last two decades the appearance of new user facilities based on $\mathrm{THz}$ free electron lasers (FELs) emitting high power short $\mathrm{THz}$ pulses provides the unique opportunity for studying of impurity centers in semiconductors under resonant excitation of chosen energy levels using single color pumpprobe technique [2-4]. In particular, the lifetimes of different donors and acceptors in silicon have been measured to localize the time scale of the existing relaxation rates. More recently similar experiments have been performed to explore impurities in germanium. Here the authors present a review of pumpprobe experiments devoted to lifetime measurements of excited donor states in silicon and germanium under the uniaxial stress.

As well known the relaxation of shallow donor electrons in semiconductors under low temperatures is governed by the emission of acoustic or optical phonons. The elemental semiconductors such as silicon and germanium have multi-valley structure of the conduction band that, together with appropriate energy gaps between states of the Coulomb centers, allow intervalley electron scattering processes. In silicon those are accompanied by the emission of f-type or gtype optical or acoustic phonons. The former corresponds to e.g. [100]-[010] transition while the latter to $[100]-[-100]$ transition in the reciprocal space. The uniaxial stress applied along [100] crystal axis removes the degeneracy resulting in splitting of valleys and corresponding energy levels. Thus uniaxial stress can modify relaxation rates if changes the energy gaps. Fig. 1 demonstrates the energy diagram of phosphorus donor in silicon under the stress as an example. The excited states move parallel to their "parent" valleys while the singlet ground state $1 \mathrm{~s}\left(\mathrm{~A}_{1}\right)$, which contains equal contributions from 6 valley under zero stress, moves nonlinearly losing the contribution of upper valleys.

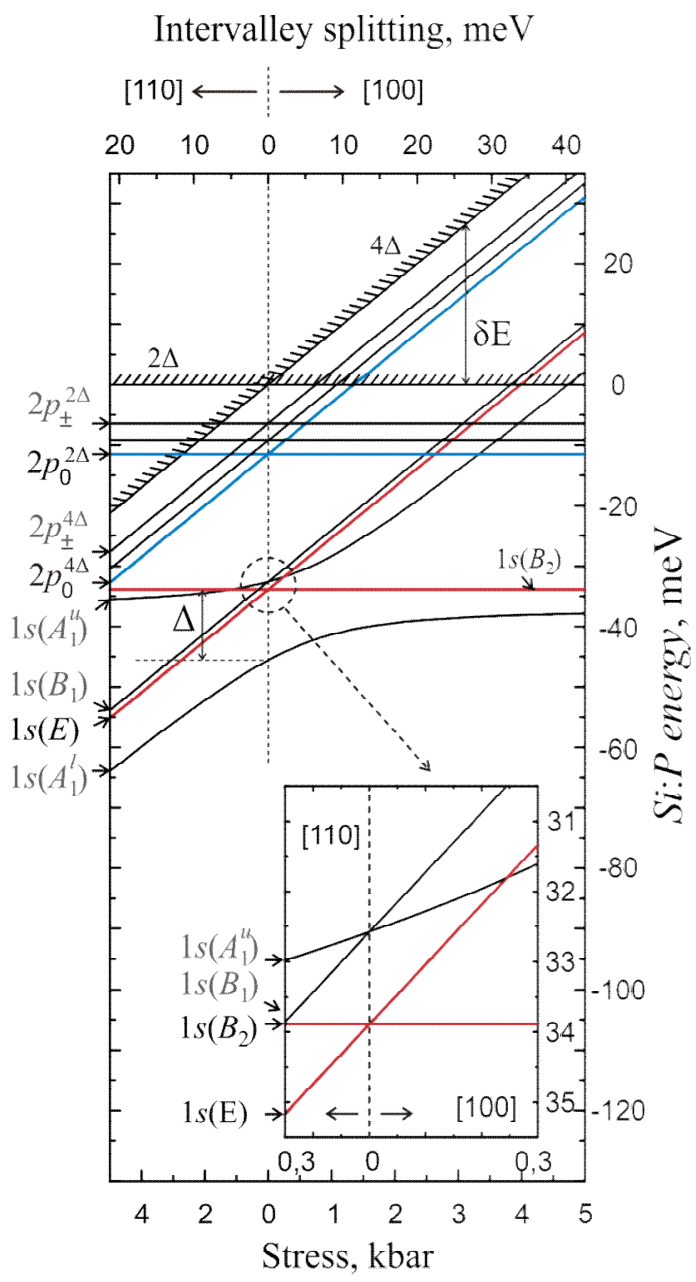

Fig. 1. Energy diagram for phosphorus donor in silicon as a function of uniaxial stress along [110] (left) or [100] (right) crystallographic orientation. Only lower excited states and ground states are shown as well as position of conduction band valleys $2 \Delta$ and $4 \Delta$. 
The silicon and germanium crystals doped by different donors were taken for sample preparation. The silicon samples were cut along 100 axis while germanium samples were cut along 111 axis. Those directions were chosen to provide the maximum valley splitting for given stress value. Typical donor concentrations were in the range of $\mathrm{N}_{\mathrm{D}}=1-5 \times 10^{15} \mathrm{~cm}^{-3}$. The samples were shaped into parallelepipeds with dimensions of $\sim 7 \times 5 \times 2 \mathrm{~mm}^{3}$. Each sample was placed into the special module designed to apply uniaxial stress. The stress module containing one sample and individual stress value was mounted in an optical liquid helium flow cryostat and cooled to about $5 \mathrm{~K}$. The achieved stress value in each module was estimated from the splitting of the donor levels as measured by absorption spectroscopy. Relaxation times of particular electronic states were derived from the timeresolved pump-probe technique. This involved the frequency-selective excitation of the investigated sample with an optical pulse, where the excitation photon energy corresponded to the energy of the transition between the probed electronic states. To measure the characteristic decay time $\tau$, the pulse duration of optical excitation must be much shorter than $\tau$ and the time delay between pump and probe pulses must be appropriate to resolve $\tau$. The overall data set was obtained using pump-probe experimental setups at several free electron laser facilities: FELIX (The Netherlands), FELBE (Germany) and NovoFEL (Russia). Details of the pump-probe setups are given elsewhere. Spectral width of the FEL emission on half width on half maximum (FWHM) was better than $1 \%$ of a FEL wavelength. The typical micropulse duration was about 10 ps for FELIX and FELBE and 100 ps for NovoFEL.

Fig. 2 demonstrates the pump-probe signals obtained for Si:P stressed along [100] when pumped into the lower stress component of $2 \mathrm{p}_{0}$ level for two values of uniaxial stress -80 and 650 bar. It is clear seen from the picture that higher stress value results in longer lifetime indicating suppression of the f-type phonon emission. Similar results have been obtained for other donor in silicon such as bismuth and arsenic. It has been obtained that typical lifetimes for donors in germanium are much longer (0.5-3 ns) than for acceptors in germanium (150-250 ps) as well as donors (100-200 ps) and acceptors (30-100 ps) in silicon. The stress applied along [111] axis for $\mathrm{Ge}: \mathrm{Sb}$ results in increase of lifetime for $2 \mathrm{p}_{ \pm}$level from $1.8 \mathrm{~ns}$ to about $3.2 \mathrm{~ns}$ that can be explained by switching off the intervalley relaxation.

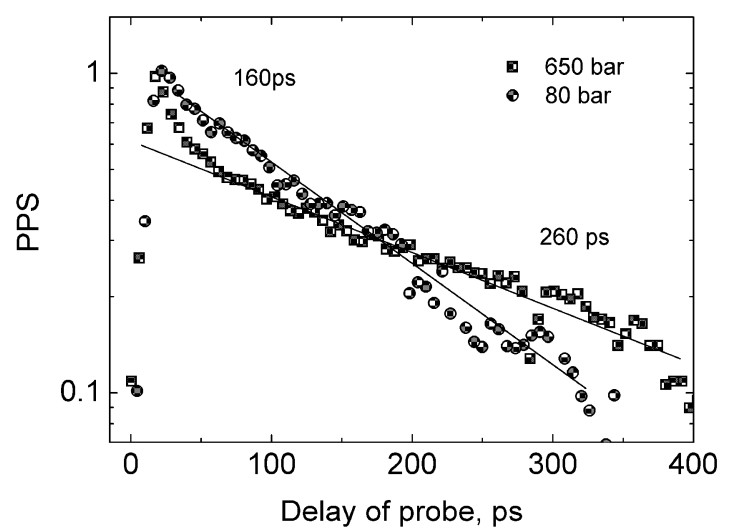

Fig. 2. Pump-probe signal for Si:P under two values of uniaxial stress (80 bar and 650 bar) along [100].

This work was partly supported by the joint GermanRussian project of the Russian Foundation for Basic Research (RFBF Project No. 18-42-520064, 18-50212077-DFG) and of the Deutsche Forschungsgemeinschaft (DFG No. 389056032).

\section{References}

1. Ramdas, A. K., Rodriguez, S. Spectroscopy of the solid-state analogues of the hydrogen atom: donors and acceptors in semiconductors, Rep. Prog. Phys. 1981, V. 4, P. 1291-1387.

2. Hübers, H.-W., Pavlov, S. G., Lynch, S. A., Greenland, Th., Litvinenko, K. L., Murdin, B., Redlich, B., van der Meer, A. F. G., Riemann, H., Abrosimov, N. V., Becker, P., Pohl, H.-J., Zhukavin, R. Kh., Shastin, V. N. Isotope effect on the lifetime of the $2 \mathrm{p}_{0}$ state in phosphorus-doped silicon // Phys. Rev. B. 2013. V. 88, P. 035201(1-5).

3. Pavlov, S. G., Deßmann, N., Pohl, A., Shuman, V. B., Portsel, L. M., Lodygin, A. N., Astrov, Yu. A., Winnerl, S., Schneider, H., Stavrias N., van der Meer, A. F. G., Tsyplenkov V. V., Kovalevsky, K. A., Zhukavin, R. Kh., Shastin, V. $N$., Abrosimov, N. V., Hübers, H.-W. Dynamics of nonequilibrium electrons on neutral center states of interstitial magnesium donors in silicon // Physical Review B. 2016. V. 94, P. 075208(1-8).

4. Choporova, Yu. Yu., Gerasimov, V. V., Knyazev, B. A., Sergeev, S. M., Shevchenko, O. A., Zhukavin, R. Kh., Abrosimov, N. V., Kovalevsky, K. A., Ovchar, V. K., Hübers, H-W., Kulipanov, G. N., Shastin, V. N., Schneider, H., Vinokurov, N. A., Kulipanov, G. N. First terahertz-range experiments on pump-probe setup at Novosibirsk free electron laser // 2016. Physics Procedia. V. 84, P. 152-156. 